\title{
THE RELATIVE VIABILITY OF HUMAN SPERMATOZOA
}

\author{
By A. C. CROOKE AND ANITA M. MANDL \\ From United Birmingham Hospitals and the Department of Anatomy, Medical School, \\ Birmingham
}

\author{
With a Statistical Analysis by Dr J. O. IRwin, M.R.C., Statistical Research Unit, \\ London School of Hygiene and Tropical Medicine
}

(With 2 Figures in the Text)

The quantitative observation of the motility of spermatozoa is not sufficiently accurate to allow a satisfactory analysis of the survival rate of the normal and the various types of abnormal spermatozoa. For this purpose, it is necessary to use fixed preparations stained with a supravital stain. This technique permits differentiation and classification of abnormal spermatozoa, indicating at the same time the proportion of cells which were alive or dead when the stain was applied.

\section{MATERIALS AND METHODS}

The material was obtained from eleven men attending an Infertility Clinic. Co-operative patients with counts of over $80 \mathrm{million} / \mathrm{ml}$. were selected. In some instances it is likely that the wife was the infertile partner and the husband was normal, but in others the husband was probably abnormal since four of them showed counts of over $20 \%$ abnormal forms. The data were based on eighteen samples of semen. One patient provided four samples, four two samples and the other six one each.

The samples were all stained by the supravital staining method described previously (Crooke \& Mandl, 1947), in which dead spermatozoa are stained blue and living ones counterstained red. The smears were examined with a $\frac{1}{12}$ in. oil immersion objective, and a fixed number (1000 from one case and 500 from each of the others) were counted and classified into normal and eight groups of abnormal forms. This classification (Crooke \& Mandl, 1948) is shown in Fig. 1. The fourteen types of abnormal forms have been grouped morphologically, except for group 8 which contains five unrelated types; these were placed into one group because they were always found to be dead. Only very small numbers of spermatozoa fall into groups 3 and 4.

For each type, counts of living and dead spermatozoa were made at 3,8 and $27 \mathrm{hr}$. after the samples were produced.

\section{RESULTS}

\section{(1) Distribution of types}

Differences in the distribution of the total numbers of each type were first examined in repeated samples from the same person. $\chi^{2}$ values were calculated for each person providing replicates (i) for variations in the proportion normal, (ii) for variations in the distribution of abnormal, and (iii) for variations in the distribution of all types. Table 1 shows the results. Generally speaking, the distribution of types varied more from one sample to another in the same person than would be expected by chance. Exceptions occur at 8 and $27 \mathrm{hr}$. in cases 4 and 7, and in all instances in case 8.

There were also significant differences in the distribution of the total numbers in each type from one person to another. Table 2 shows the values of $\chi^{2}$ obtained. The significance is very great in both cases, but the change in proportion of normals is much more striking than in the relative numbers of abnormal forms among themselves. In view of this, an unweighted mean of the percentages at each count was calculated for each person.

Table 3A shows the results for all types, and Table $3 \mathrm{~B}$ for the abnormal ones only. The counts at the three different times are remarkably consistent. The difference between case 5 and the others is particularly marked.

\section{(2) Mortalities in normal and abnormal spermatozoa}

For each type of spermatozoa the mortalities (i) in different samples from the same person, (ii) in different persons, and (iii) in all samples, were tested for significant differences by calculating $x^{2}$ values from the formula

$$
\chi^{2}=\frac{\Sigma n(p-P)^{2}}{P Q},
$$

where $p$ is the mortality for an individual sample and $P$ is the overall mortality (Table 4). In different 
samples from the same person, normal spermatozoa show greater differences in mortality than would be expected by chance. The same is true for type 8 in two cases ( 3 and $8 \mathrm{hr}$.) and for type 2 in two cases (8 and $27 \mathrm{hr}$.). In addition, there are sometimes significantly greater differences in mortality between persons than would be expected from the replicates.
The differences in mortality between the different types are also remarkably consistent. The normals have the lowest mortality, and type 8 the highest. The order of increasing mortality is 1 , then $2,3,4,5$ and 6 all about the same, 7 somewhat higher, then 8 (Fig. 2). No stress can be laid on the values for types 3 and 4, owing to the small numbers.

Abnormal forms
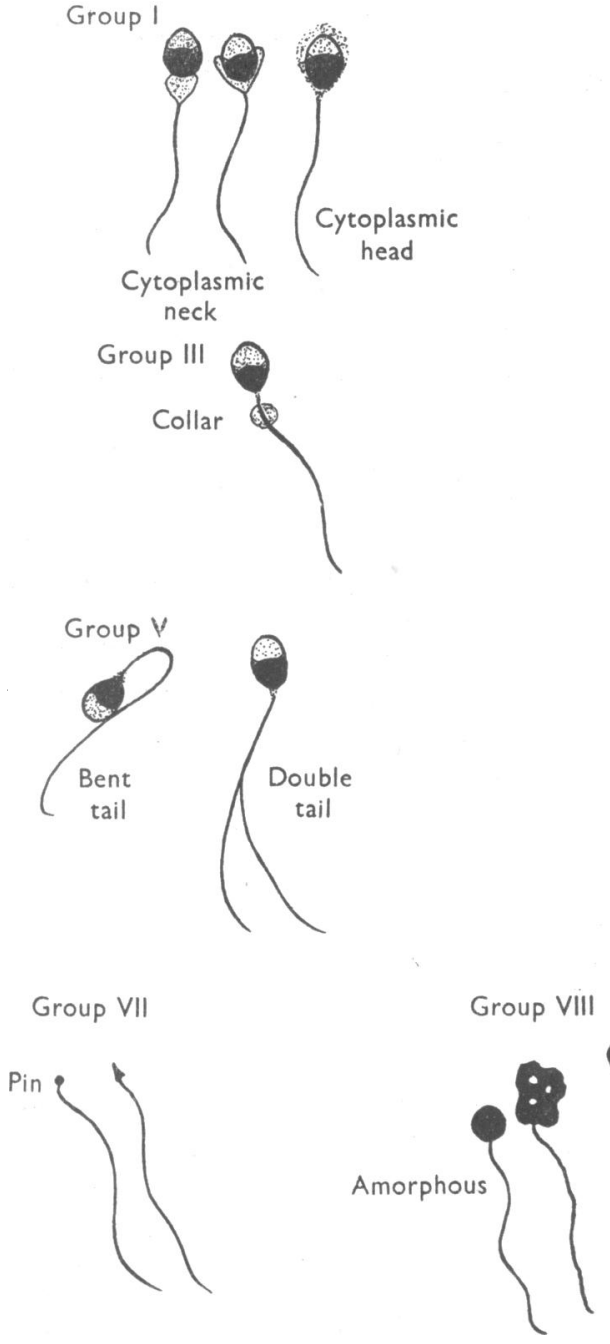

Fig. 1. Classification of abnormal spermatozoa into fourteen types and eight groups.

This is true for type 1 at 8 and $27 \mathrm{hr}$., for type 6 at $27 \mathrm{hr}$., and for type 7 at $27 \mathrm{hr}$.: where the results are individually not significant, the tendency is still in the same direction.

For this reason unweighted means of the mortalities for each type and person have been calculated and are shown in Table 5.
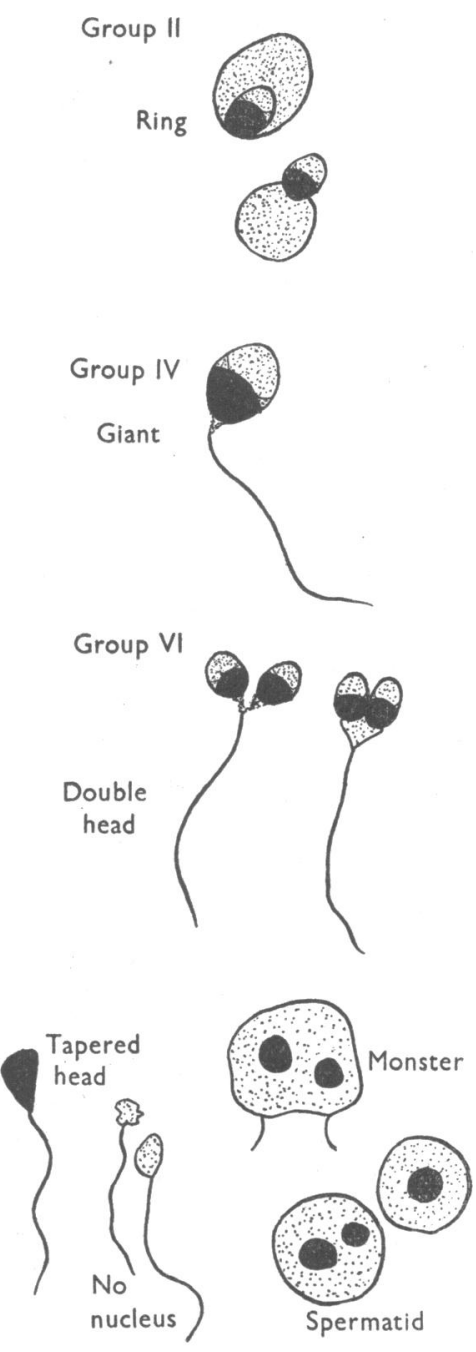

\section{DISCUSSION}

The validity of the results described in this paper depends on whether the stain can be relied upon to differentiate dead from living cells by staining all the dead cells and neither colouring or killing the living ones. 
Table 1. Distribution of types. $\chi^{2}$ and degrees of freedom

Normal $v$. abnormal

Between different types of abnormal

Between all types

Normal $v$. abnormal

Between different types of abnormal

Between all types

Normal $v$. abnormal

Between different types of abnormal

Between all types
Case 4

Caso

3 hours

$37 \cdot 32$ (1)

Case 6

Case 7

Case 8

$10 \cdot 60(1)$
$22 \cdot 41(5)$

$40.95(3)$

$5 \cdot 30(1)$

$0 \cdot 12(1)$

$38 \cdot 27$ (5)

$\mathbf{8 9 \cdot 0 5}(15)$

$17 \cdot 54(5)$

$9 \cdot 49(5)$

$33 \cdot 01(6)$

$75 \cdot 59(6)$

$131 \cdot 91(18)$

$22 \cdot 84(6)$

$9 \cdot 61(6)$

8 hours

\begin{tabular}{|c|c|c|c|c|}
\hline $\begin{array}{r}3 \cdot 57 \\
20 \cdot 12\end{array}$ & $\begin{array}{l}19 \cdot 60 \\
23 \cdot 61\end{array}$ & $\begin{array}{l}33 \cdot 03 \\
70 \cdot 78\end{array}$ & $\begin{array}{l}5 \cdot 35 \\
7 \cdot 73\end{array}$ & $\begin{array}{c}0.20 \\
7 \cdot 44 \\
.\end{array}$ \\
\hline $23 \cdot 70$ & $43 \cdot 21$ & $113 \cdot 29$ & $13 \cdot 08$ & $7 \cdot 64$ \\
\hline \multicolumn{5}{|c|}{27 hours } \\
\hline $\begin{array}{r}0 \cdot 006 \\
15 \cdot 264\end{array}$ & $\begin{array}{r}9.91 \\
36.64\end{array}$ & $\begin{array}{l}15 \cdot 53 \\
85 \cdot 30\end{array}$ & $\begin{array}{l}1 \cdot 78 \\
3 \cdot 40\end{array}$ & $\begin{array}{r}0.41 \\
10.08\end{array}$ \\
\hline $15 \cdot 27$ & $46 \cdot 55$ & $105 \cdot 01$ & $5 \cdot 18$ & $10 \cdot 49$ \\
\hline
\end{tabular}

Values significant at the $5 \%$ level are in black.

To facilitate calculation, groups 2, 3 and 4 have been treated as one group.

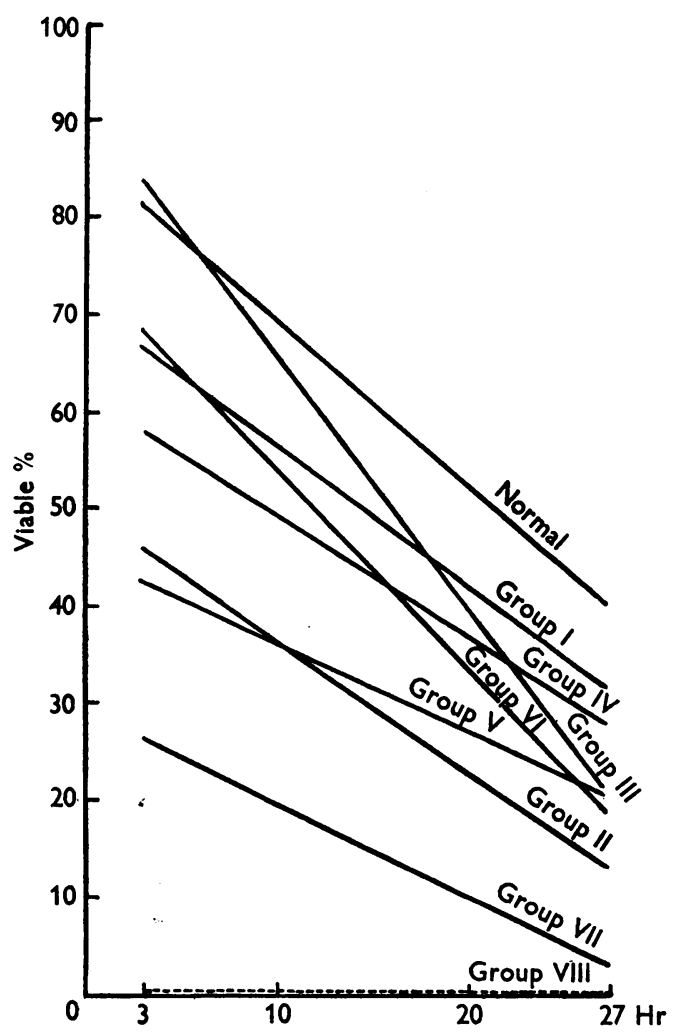

Fig. 2. Mortality slopes of normal and eight groups of abnormal spermatozoa, based on a count of 28,500 cells.

The reliability of the stain was demonstrated in three different ways. First, semen was mixed with buffered soluble blue, then centrifuged, and the spermatozoa resuspended in Baker's fluid. All motile spermatozoa were found to be colourless and the nonmotile ones were mostly stained blue. The probable reason why a few non-motile spermatozoa cannot be stained is that they are living (Emmens, 1947). A stained motile cell was never seen.

Next it was observed that the number of sperma. tozoa which took the supravital stain increased with the age of the sample and that this increase was related to decreasing motility. Emmens (1947) reported similar findings with rabbit semen.

Table 2. Values of $\chi^{2}$ for significance of change in distribution of types from person to person

\begin{tabular}{rccccc}
$\chi^{2}$ & $\begin{array}{c}\text { Degrees of } \\
\text { freedom }\end{array}$ & $\overbrace{\chi^{2}}^{\text {Normal } v \text { abnormal }}$ & \multicolumn{1}{c}{$\begin{array}{c}\text { Degrees of } \\
\text { freedom }\end{array}$} \\
$3 \mathrm{hr}$. & 1181 & 10 & 613 & 50 \\
$8 \mathrm{hr}$. & 1467 & 10 & 612 & 50 \\
$27 \mathrm{hr}$. & 1352 & 10 & 658 & 50
\end{tabular}

To facilitate calculation, groups 2,3 and 4 have been calculated as one group.

Finally, some seminal fluid was mixed with the stain and left at room temperature for $36 \mathrm{hr}$. Drops were withdrawn at intervals, examined for motility and compared with an unstained control derived from the same specimen. It was found that the motility of the stained and unstained fractions was identical, and a few moving spermatozoa were observed at the end of $36 \mathrm{hr}$.

We felt justified, therefore, in analysing statistically the viability of normal and the various types of abnormal spermatozoa. There is close agreement 
between various counts made at different times on the same sample, both in the classification of abnormal forms and the estimation of the relative viability of different forms. Perusal of individual counts (Table $3 \mathrm{~A}$ ) bears this out.

The statistical analysis of the present results

Table 3 A. Percentage distribution of types

Abnormal

\begin{tabular}{|c|c|c|c|c|c|c|c|c|c|}
\hline \multicolumn{2}{|c|}{ Case Normal } & 11 & 2 & 3 & 4 & 5 & 6 & 7 & 8 \\
\hline \multicolumn{10}{|c|}{$3 \mathrm{hr}$. } \\
\hline 1 & $84 \cdot 0$ & $7 \cdot 6$ & $1 \cdot 8$ & $0 \cdot 0$ & $0 \cdot 3$ & $0 \cdot 6$ & $1 \cdot 3$ & $2 \cdot 3$ & $2 \cdot 1$ \\
\hline 2 & $87 \cdot 6$ & $4 \cdot 8$ & $2 \cdot 0$ & 0.0 & $0 \cdot 6$ & 0.2 & $0 \cdot 2$ & 0.2 & $4 \cdot 4$ \\
\hline 3 & $87 \cdot 4$ & $6 \cdot 6$ & $1 \cdot 4$ & 0.0 & $0 \cdot 4$ & $1 \cdot 4$ & 0.4 & 0.2 & $2 \cdot 2$ \\
\hline 4 & $80 \cdot 5$ & $4 \cdot 7$ & $\mathbf{3} \cdot \mathbf{3}$ & $0 \cdot 1$ & $1 \cdot 3$ & $1 \cdot 5$ & 0.9 & $2 \cdot 6$ & $5 \cdot 1$ \\
\hline 5 & $36 \cdot 5$ & $19 \cdot 6$ & $29 \cdot 8$ & $0 \cdot 1$ & 0.0 & $3 \cdot 0$ & $4 \cdot 1$ & 0.5 & $6 \cdot 4$ \\
\hline 6 & $73 \cdot 0$ & $8 \cdot 6$ & $5 \cdot 75$ & 0.05 & 0.4 & $3 \cdot 9$ & 0.5 & $1 \cdot 3$ & $6 \cdot 6$ \\
\hline 7 & $85 \cdot 4$ & $6 \cdot 1$ & $3 \cdot 6$ & $0 \cdot 2$ & 0.4 & $0 \cdot 6$ & $1 \cdot 0$ & 0.5 & $2 \cdot 2$ \\
\hline 8 & $85 \cdot 8$ & $5 \cdot 4$ & $2 \cdot 0$ & $0 \cdot 1$ & 0.4 & $2 \cdot 3$ & 0.4 & $0 \cdot 2$ & $3 \cdot 4$ \\
\hline 9 & $64 \cdot 0$ & $6 \cdot 4$ & $28 \cdot 6$ & $0 \cdot 2$ & 0.0 & 0.0 & $0 \cdot 4$ & 0.0 & 0.4 \\
\hline 10 & $89 \cdot 0$ & $4 \cdot 0$ & $5 \cdot 0$ & 0.0 & $0 \cdot 2$ & $0 \cdot 4$ & 0.2 & 0.0 & $1 \cdot 2$ \\
\hline 11 & $81 \cdot 2$ & $11 \cdot 6$ & $5 \cdot 0$ & 0.0 & 0.0 & $1 \cdot 0$ & $0 \cdot 2$ & 0.0 & $1 \cdot 0$ \\
\hline Av. & $77 \cdot 7$ & $7 \cdot 8$ & $7 \cdot 8$ & 0.7 & 0.36 & $1 \cdot 4$ & 0.9 & 0.7 & 3.2 \\
\hline
\end{tabular}

8 hr.

$\begin{array}{llllllllll}\text { l } & 85.9 & 7.9 & 1.2 & 0.3 & 0.2 & 0.3 & 0.4 & 1.6 & 2.2\end{array}$

$\begin{array}{lllllllllll}2 & 89.8 & 4.6 & 1.6 & 0.0 & 0.8 & 0.8 & 0.2 & 0.0 & 2.2\end{array}$

$\begin{array}{llllllllll}3 & 86.6 & 6.6 & 1.8 & 0.0 & 0.2 & 2.0 & 0.0 & 0.4 & 2.4\end{array}$

$\begin{array}{llllllllll}4 & 82.5 & 4.4 & 3.0 & 0.0 & 0.7 & 1.0 & 0.6 & 2.7 & 5.0\end{array}$

$\begin{array}{lllllllllll}5 & 36.3 & 19.4 & 31.3 & 0.1 & 0.6 & 2.9 & 3.5 & 0.8 & 5.5\end{array}$

$\begin{array}{llllllllll}6 & 73.0 & 8.2 & 5.8 & 0.0 & 1.0 & 4.0 & 0.9 & 1.2 & 6.5\end{array}$

$\begin{array}{llllllllll}7 & 86.5 & 5.8 & 3.5 & 0.0 & 0.3 & 0.2 & 0.7 & 0.2 & 2.8\end{array}$

$\begin{array}{llllllllll}8 & 85 \cdot 1 & 5 \cdot 1 & 1 \cdot 6 & 0 \cdot 0 & 0 \cdot 0 & 3 \cdot 0 & 1 \cdot 1 & 0 \cdot 4 & 3 \cdot 7\end{array}$

$\begin{array}{llllllllll}9 & 62.0 & 7.6 & 28.0 & 0.0 & 0.0 & 0.0 & 0.8 & 0.4 & 1.2\end{array}$

$\begin{array}{llllllllll}10 & 91.8 & 2.6 & 4.0 & 0.0 & 0.2 & 0.2 & 0.0 & 0.0 & 1.2\end{array}$

$\begin{array}{llllllllll}11 & 80.0 & 11.4 & 4.0 & 0.0 & 1.0 & 0.2 & 0.2 & 0.2 & 3.0\end{array}$

$\begin{array}{llllllllll}\text { Av. } & 78.1 & 7.6 & 7.8 & 0.36 & 0.45 & 1.3 & 0.8 & 0.7 & 3.2\end{array}$

$27 \mathrm{hr}$.

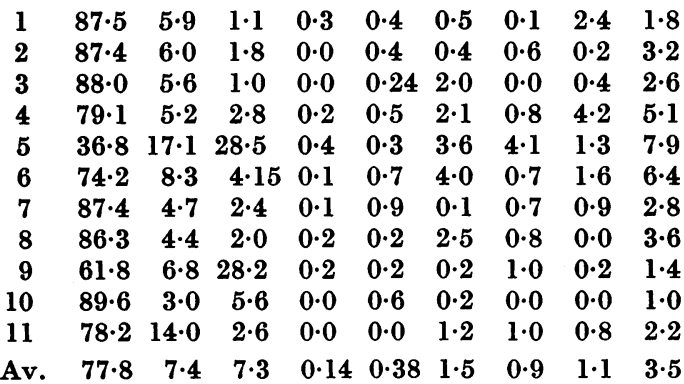

shows that the series of eighteen samples derived from eleven patients is a heterogeneous one. The proportion of normal and various types of abnormal cells varied significantly both between the eleven donors, and between different samples provided by the same donor. The rate of death, similarly, varies significantly from patient to patient. Nevertheless, the results for different patients are sufficiently consistent to show that the differences in mortality between type 1, type 8 and the remaining types are significant.

The mortality slopes are all more or less parallel, though starting at somewhat different levels at $3 \mathrm{hr}$. This is true for all types, except tapered head, amorphous, no nucleus, monster and spermatid,

Table 3 B. Percentage distribution of abnormal types

\begin{tabular}{|c|c|c|c|c|c|c|c|c|}
\hline \multirow[b]{2}{*}{ Case } & \multicolumn{8}{|c|}{ Type } \\
\hline & 1 & 2 & 3 & 4 & 5 & 6 & 7 & 8 \\
\hline \multicolumn{9}{|c|}{$3 \mathrm{hr}}$. \\
\hline 1 & $47 \cdot 5$ & $10 \cdot 6$ & 0 & $2 \cdot 5$ & $3 \cdot 8$ & 8.4 & $14 \cdot 4$ & \\
\hline 2 & 38.7 & $16 \cdot 1$ & 0 & & 1.6 & & & \\
\hline 3 & $52 \cdot 4$ & $11 \cdot 0$ & 0 & $3 \cdot 3$ & $11 \cdot 1$ & $3 \cdot 2$ & 1.6 & 17 \\
\hline 4 & $23 \cdot 1$ & $17 \cdot 1$ & 0.5 & & 8.9 & 8 & $12 \cdot 2$ & 26 \\
\hline 5 & $30 \cdot 1$ & 46.9 & & 0 & 4.7 & 1 & & \\
\hline 6 & $31 \cdot 4$ & $21 \cdot 3$ & 15 & $1 \cdot 43$ & $15 \cdot 6$ & $\cdot 8$ & & 23 \\
\hline 7 & 40.5 & $24 \cdot 65$ & 1 . & $2 \cdot 7$ & $4 \cdot 3$ & & & 17 \\
\hline 8 & $38 \cdot 4$ & $14 \cdot 0$ & & 2 . & & .8 & & 23 \\
\hline 9 & $17 \cdot 8$ & $79 \cdot 44$ & & 0 & & & & \\
\hline 10 & 36.4 & 45.4 & 0 & 1.8 & & 1.8 & & \\
\hline 11 & 61.7 & $26 \cdot 6$ & 0 & 0 & & $1 \cdot 1$ & & \\
\hline Av. & $38 \cdot 0$ & $28 \cdot 5$ & $0 \cdot 82$ & $2 \cdot 4$ & $6 \cdot 8$ & $3 \cdot 5$ & $\cdot 6$ & 16 \\
\hline \multicolumn{9}{|c|}{$8 \mathrm{hr}$. } \\
\hline 1 & $56 \cdot 0$ & 8.5 & & & $2 \cdot 1$ & $2 \cdot 8$ & $11 \cdot 3$ & 15 \\
\hline 2 & $45 \cdot 1$ & 15.4 & 0 & & & $2 \cdot 0$ & & \\
\hline 3 & $49 \cdot 3$ & $11 \cdot 6$ & 0 & & $14 \cdot 9$ & 0 & $\cdot 0$ & 17 \\
\hline 4 & $25 \cdot 7$ & $17 \cdot 5$ & & 4 & & $3 \cdot 1$ & $14 \cdot 8$ & 28 \\
\hline 5 & $29 \cdot 9$ & 49. & 0.2 & 1 . & & $5 \cdot 3$ & $1 \cdot 1$ & \\
\hline 6 & 30.4 & 21 . & 0 & & $14 \cdot 6$ & 3. & $\cdot 9$ & 23 \\
\hline 7 & $42 \cdot 8$ & 25 . & 0 & $2 \cdot 2$ & 1.5 & & $\cdot 3$ & \\
\hline 8 & $34 \cdot 0$ & & 0 & 0 & $20 \cdot 3$ & 7. & .7 & 24 \\
\hline 9 & $20 \cdot 0$ & 73 & 0 & & 0 & $2 \cdot 1$ & $\cdot 1$ & \\
\hline 10 & $31 \cdot 7$ & $48 \cdot 8$ & 0 & $2 \cdot 4$ & $2 \cdot 4$ & 0 & & 14 \\
\hline 11 & $57 \cdot 0$ & $20 \cdot$ & 0 & 5 & 1.0 & $1 \cdot 0$ & $1 \cdot 0$ & 15 \\
\hline Av. & $38 \cdot 3$ & $27 \cdot 5$ & 0.21 & $2 \cdot 6$ & $6 \cdot 9$ & 3.0 & $3 \cdot 6$ & 17 \\
\hline \multicolumn{9}{|c|}{$27 \mathrm{hr}$. } \\
\hline 1 & $47 \cdot 2$ & 8 & $2 \cdot 4$ & 3.2 & $4 \cdot 0$ & & & \\
\hline 2 & $47 \cdot$ & $14 \cdot 3$ & 0 & & & & & \\
\hline 3 & $46 \cdot 7$ & 8.4 & 0 & 3. & $16 \cdot 7$ & 0 & $\mathbf{3} \cdot \mathbf{3}$ & \\
\hline 4 & 24.9 & $13 \cdot 4$ & 0.95 & $2 \cdot 4$ & $10 \cdot 1$ & $3 \cdot 8$ & $20 \cdot 1$ & \\
\hline 5 & 26 . & & & 0.45 & & & & \\
\hline 6 & 31. & 16 & 0 & 2 & $16 \cdot 3$ & 2 & & 24 \\
\hline 7 & 37. & 19. & & & & & $7 \cdot 1$ & \\
\hline 8 & $31 \cdot 6$ & & & & 18. & 5 & 0 & \\
\hline 9 & $17 \cdot 8$ & $73 \cdot$ & 0.5 & 0.5 & & $2 \cdot 6$ & & \\
\hline 10 & 28.8 & & 0 & & & 0 & 0 & \\
\hline 11 & $64 \cdot 2$ & & 0 & 0 & 5.5 & $4 \cdot 6$ & $3 \cdot 7$ & \\
\hline Av. & $36 \cdot 8$ & $25 \cdot 4$ & 0.26 & 2.7 & $7 \cdot 6$ & $3 \cdot 3$ & $5 \cdot 7$ & 17 \\
\hline
\end{tabular}

which are always dead. These results indicate a somewhat smaller chance of survival amongst the abnormal types; nevertheless, their survival time is long enough to suggest that though they are abnormal in structure, they would be capable of performing normal metabolic functions. Whether or not they are able to fertilize an ovum requires an experimental investigation, but at present there seems to be no reason to preclude this possibility. 


\begin{tabular}{|c|c|c|c|c|c|c|c|c|}
\hline & \multirow[b]{2}{*}{ Normal } & \multicolumn{6}{|c|}{ Abnormal } & \multirow[b]{2}{*}{$\begin{array}{c}\text { Degrees of } \\
\text { freedom }\end{array}$} \\
\hline & & 1 & $\begin{array}{c}2,3 \\
\text { and } 4 \\
3\end{array}$ & $\begin{array}{r}5 \\
\text { ours }\end{array}$ & 6 & 7 & 8 & \\
\hline Between persons & $268 \cdot 23$ & $26 \cdot 09$ & $57 \cdot 55$ & $16 \cdot 34$ & $7 \cdot 89$ & $6 \cdot 58$ & $10 \cdot 62$ & 10 \\
\hline Within persons & $87 \cdot 07$ & $9 \cdot 56$ & $12 \cdot 79$ & $12 \cdot 18$ & $9 \cdot 47$ & $11 \cdot 02$ & $20 \cdot 48$ & 7 \\
\hline \multirow[t]{2}{*}{ Total } & $355 \cdot 3$ & $35 \cdot 65$ & $70 \cdot 35$ & $28 \cdot 52$ & $17 \cdot 36$ & $17 \cdot 60$ & $31 \cdot 10$ & 17 \\
\hline & \multicolumn{7}{|c|}{8 hours } & \\
\hline Between persons & $252 \cdot 00$ & $114 \cdot 33\}$ & $44 \cdot 96$ & $18 \cdot 04$ & $15 \cdot 97$ & $4 \cdot 54$ & $14 \cdot 13$ & 10 \\
\hline Within persons & $128 \cdot 70$ & $13.03\}$ & $15 \cdot 74$ & $6 \cdot 24$ & $3 \cdot 05$ & $3 \cdot 30$ & $35 \cdot 14$ & 7 \\
\hline \multirow[t]{2}{*}{ Total } & $380 \cdot 70$ & $127 \cdot 36$ & $60 \cdot 70$ & $24 \cdot 28$ & $19 \cdot 02$ & $7 \cdot 84$ & $49 \cdot 27$ & 17 \\
\hline & \multicolumn{7}{|c|}{27 hours } & \\
\hline Between persons & $816 \cdot 38$ & $82 \cdot 20\}$ & $29 \cdot 65$ & $23 \cdot 83$ & $\mathbf{3 1} \cdot \mathbf{2 4}\}$ & $6.95)$ & 3.94 & 10 \\
\hline Within persons & $181 \cdot 51$ & $13.41\}$ & $26 \cdot 36$ & 3.99 & $3.01\}$ & $0 \cdot 84\}$ & $5 \cdot 92$ & 7 \\
\hline Total & $997 \cdot 89$ & $95 \cdot 61$ & $56 \cdot 01$ & $27 \cdot 82$ & $34 \cdot 25$ & $7 \cdot 79$ & $9 \cdot 86$ & 17 \\
\hline
\end{tabular}

Table 5. Percentage mortalities by type and case

Abnormal

\begin{tabular}{|c|c|c|c|c|c|c|c|c|c|}
\hline Case & Normal & 1 & 2 & \multicolumn{2}{|c|}{3 hours } & 5 & 6 & 7 & 8 \\
\hline 1 & $23 \cdot 9$ & $34 \cdot 2$ & $55 \cdot 5$ & - & $33 \cdot 3$ & $50 \cdot 0$ & $53 \cdot 9$ & $65 \cdot 2$ & $100 \cdot 0$ \\
\hline 2 & $28 \cdot 1$ & $37 \cdot 5$ & $50 \cdot 0$ & - & $100 \cdot 0$ & $100 \cdot 0$ & 0 & $100 \cdot 0$ & $100 \cdot 0$ \\
\hline 3 & $20 \cdot 4$ & $33 \cdot 3$ & $71 \cdot 4$ & - & $50 \cdot 0$ & $28 \cdot 6$ & $50 \cdot 0$ & $100 \cdot 0$ & $100 \cdot 0$ \\
\hline 4 & $19 \cdot 1$ & $21 \cdot 3$ & $57 \cdot 5$ & 0 & $42 \cdot 9$ & $72 \cdot 7$ & $66 \cdot 7$ & 62.9 & $100 \cdot 0$ \\
\hline 5 & $27 \cdot 3$ & $44 \cdot 1$ & $63 \cdot 7$ & $100 \cdot 0$ & - & $77 \cdot 8$ & $32 \cdot 4$ & $100 \cdot 0$ & $97 \cdot 8$ \\
\hline 6 & $28 \cdot 1$ & $44 \cdot 1$ & $68 \cdot 7$ & 0 & $44 \cdot 4$ & $63 \cdot 5$ & $58 \cdot 3$ & $69 \cdot 3$ & $96 \cdot 9$ \\
\hline 7 & $6 \cdot 7$ & $24 \cdot 7$ & $25 \cdot 0$ & 0 & $33 \cdot 3$ & $16 \cdot 7$ & $20 \cdot 0$ & $40 \cdot 0$ & $100 \cdot 0$ \\
\hline 8 & $15 \cdot 5$ & $47 \cdot 9$ & $65 \cdot 0$ & 0 & $33 \cdot 3$ & $71 \cdot 3$ & $16 \cdot 7$ & $50 \cdot 0$ & $100 \cdot 0$ \\
\hline 9 & 12.5 & $28 \cdot 1$ & $35 \cdot 0$ & $100 \cdot 0$ & - & - & $50 \cdot 0$ & - & $100 \cdot 0$ \\
\hline 10 & $20 \cdot 2$ & $30 \cdot 0$ & $60 \cdot 0$ & - & 0 & $50 \cdot 0$ & 0 & - & $100 \cdot 0$ \\
\hline 11 & $6 \cdot 4$ & $20 \cdot 7$ & $68 \cdot 0$ & - & - & $40 \cdot 0$ & 0 & - & $100 \cdot 0$ \\
\hline Av. & $18 \cdot 9$ & $33 \cdot 3$ & $56 \cdot 3$ & $16 \cdot 6$ & $42 \cdot 1$ & $57 \cdot 1$ & $31 \cdot 6$ & $73 \cdot 4$ & $99 \cdot 5$ \\
\hline \multicolumn{10}{|c|}{8 hours } \\
\hline 1 & $40 \cdot 1$ & $45 \cdot 6$ & $75 \cdot 0$ & $100 \cdot 0$ & $50 \cdot 0$ & $33 \cdot 3$ & $50 \cdot 0$ & $75 \cdot 0$ & $100 \cdot 0$ \\
\hline 2 & $31 \cdot 6$ & $26 \cdot 1$ & 62.5 & - & $50 \cdot 0$ & $100 \cdot 0$ & 0 & 0 & $100 \cdot 0$ \\
\hline 3 & $30 \cdot 7$ & $48 \cdot 5$ & $77 \cdot 7$ & - & 0 & $50 \cdot 0$ & - & $100 \cdot 0$ & $100 \cdot 0$ \\
\hline 4 & 38.6 & $69 \cdot 9$ & $86 \cdot 7$ & - & $100 \cdot 0$ & 94.5 & $83 \cdot 3$ & $78 \cdot 6$ & $100 \cdot 0$ \\
\hline 5 & $48 \cdot 3$ & $75 \cdot 5$ & $81 \cdot 8$ & $100 \cdot 0$ & $83 \cdot 3$ & $87 \cdot 0$ & $80 \cdot 8$ & $87 \cdot 5$ & $98 \cdot 6$ \\
\hline 6 & $42 \cdot 4$ & $60 \cdot 2$ & $78 \cdot 1$ & - & $55 \cdot 0$ & $72 \cdot 6$ & $35 \cdot 7$ & $78 \cdot 0$ & $95 \cdot 8$ \\
\hline 7 & $26 \cdot 7$ & $29 \cdot 4$ & $60 \cdot 0$ & - & $66 \cdot 7$ & $50 \cdot 0$ & $41 \cdot 7$ & $50 \cdot 0$ & $100 \cdot 0$ \\
\hline 8 & $28 \cdot 1$ & $65 \cdot 5$ & $81 \cdot 2$ & - & - & $82 \cdot 0$ & $85 \cdot 7$ & $100 \cdot 0$ & $100 \cdot 0$ \\
\hline 9 & $19 \cdot 35$ & $52 \cdot 6$ & $57 \cdot 1$ & - & - & - & $75 \cdot 0$ & $100 \cdot 0$ & $100 \cdot 0$ \\
\hline 10 & $36 \cdot 4$ & $61 \cdot 5$ & $75 \cdot 0$ & - & $100 \cdot 0$ & $100 \cdot 0$ & - & - & $100 \cdot 0$ \\
\hline 11 & $11 \cdot 5$ & $14 \cdot 0$ & $70 \cdot 0$ & - & 0 & $100 \cdot 0$ & - & $100 \cdot 0$ & $100 \cdot 0$ \\
\hline Av. & $32 \cdot 2$ & $49 \cdot 9$ & $73 \cdot 2$ & $100 \cdot 0$ & $63 \cdot 1$ & $76 \cdot 9$ & $56 \cdot 5$ & $76 \cdot 9$ & $99 \cdot 5$ \\
\hline \multicolumn{10}{|c|}{27 hours } \\
\hline 1 & $58 \cdot 1$ & $67 \cdot 8$ & $81 \cdot 8$ & $100 \cdot 0$ & $75 \cdot 0$ & $100 \cdot 0$ & $100 \cdot 0$ & $83 \cdot 3$ & $100 \cdot 0$ \\
\hline 2 & $66 \cdot 6$ & $70 \cdot 0$ & $77 \cdot 7$ & - & $100 \cdot 0$ & $100 \cdot 0$ & 66.7 & $100 \cdot 0$ & $100 \cdot 0$ \\
\hline 3 & $68 \cdot 2$ & 67.9 & $100 \cdot 0$ & - & $50 \cdot 0$ & $80 \cdot 0$ & - & $100 \cdot 0$ & $100 \cdot 0$ \\
\hline 4 & 68.5 & $67 \cdot 2$ & $78 \cdot 6$ & $100 \cdot 0$ & $100 \cdot 0$ & $91 \cdot 2$ & $87 \cdot 5$ & $93 \cdot 1$ & $100 \cdot 0$ \\
\hline 5 & $58 \cdot 4$ & $68 \cdot 4$ & 83.5 & $50 \cdot 0$ & 0 & $94 \cdot 1$ & 92.9 & $100 \cdot 0$ & $100 \cdot 0$ \\
\hline 6 . & $58 \cdot 3$ & $75 \cdot 7$ & $90 \cdot 4$ & $100 \cdot 0$ & $92 \cdot 8$ & $85 \cdot 3$ & $100 \cdot 0$ & $98 \cdot 3$ & $99 \cdot 2$ \\
\hline 7 & $35 \cdot 1$ & $53 \cdot 7$ & $79 \cdot 2$ & 0 & 55.5 & 0 & $45 \cdot 0$ & $100 \cdot 0$ & $100 \cdot 0$ \\
\hline 8 & $77 \cdot 9$ & $85 \cdot 2$ & $95 \cdot 0$ & $100 \cdot 0$ & $50 \cdot 0$ & 93.8 & $100 \cdot 0$ & - & $100 \cdot 0$ \\
\hline 9 & $94 \cdot 8$ & $94 \cdot 1$ & $95 \cdot 0$ & $100 \cdot 0$ & $100 \cdot 0$ & $100 \cdot 0$ & $100 \cdot 0$ & $100 \cdot 0$ & $100 \cdot 0$ \\
\hline 10 & $46 \cdot 0$ & $80 \cdot 0$ & $96 \cdot 4$ & - & $100 \cdot 0$ & $100 \cdot 0$ & - & - & $100 \cdot 0$ \\
\hline 11 & $22 \cdot 5$ & $27 \cdot 1$ & $69 \cdot 2$ & - & - & $33 \cdot 3$ & $40 \cdot 0$ & $100 \cdot 0$ & 100.0 \\
\hline Av. & $59 \cdot 5$ & $68 \cdot 8$ & $86 \cdot 1$ & $78 \cdot 6$ & $72 \cdot 3$ & $79 \cdot 8$ & $81 \cdot 3$ & $97 \cdot 2$ & $99 \cdot 9$ \\
\hline \multicolumn{9}{|c|}{ J. Hygiene 47} & 20 \\
\hline
\end{tabular}




\section{SUMMARY}

A heterogeneous sample consisting of eighteen specimens of semen provided by eleven patients was examined. Smears were supravitally stained at 3,8 and $27 \mathrm{hr}$. Counts of living and dead sperma- tozoa, classified into normal and fourteen types of abnormal forms, showed that whereas five abnormal forms were always dead, the remaining types showed varying proportions of dead and living cells. Their rate of mortality was somewhat greater than that of normal spermatozoa.

\section{REFERENCES}

Crooke, A. C. \& Mande, A. M. (1947). Nature, Lond., $159,749$.

Crooke, A. C. \& Mande, A. M. (1948). Human Fertility.

In the Press.

Emmens, C. W. (1947). J. Physiol. 106, 471.

(MS. received for publication 19. v. 49.-Ed.) 